\title{
Erratum: All-order quartic couplings in highly symmetric D-brane-anti-D-brane systems
}

\author{
Ehsan Hatefi ${ }^{a}$ and Per Sundell ${ }^{b}$ \\ a Scuola Normale Superiore and I.N.F.N., \\ Piazza dei Cavalieri 7, 56126, Pisa, Italy \\ ${ }^{b}$ Departamento de Ciencias Físicas, Universidad Andres Bello, \\ Sazié 2212, Santiago, Chile \\ E-mail: ehsanhatefi@gmail.com, per.anders.sundell@gmail.com
}

ERRATUM TO: JHEP05(2021)245

ARXIV EPRINT: 2103.06302

Author Ehsan Hatefi has just this affiliation:

Scuola Normale Superiore and I.N.F.N., Piazza dei Cavalieri 7, 56126, Pisa, Italy

Open Access. This article is distributed under the terms of the Creative Commons Attribution License (CC-BY 4.0), which permits any use, distribution and reproduction in any medium, provided the original author(s) and source are credited. 